International Mathematical Forum, 1, 2006, no. 18, 893-895

\title{
A Simple Note about Matrices Symmetric in Signs
}

\author{
Gregory L. Light \\ Providence College, Providence \\ Rhode Island 02918 U.S.A. \\ glight@providence.edu
}

\begin{abstract}
This paper proves that any real matrix symmetric in signs, $\{+,-$, $0\}$, has no pure imaginary eigenvalues, so that if it is also of full rank, then it is hyperbolic - information useful in dynamical systems.
\end{abstract}

Mathematics Subject Classification: 15A18, 37C75, 37D10

Keywords: Sign pattern matrix, hyperbolicity, imaginary eigenvalues

\section{Introduction}

This short note is concerned with the eigenvalues of real matrices with symmetric sign patterns, which will henceforth be denoted by $H$. The study of general "sign pattern matrices" - - that is, matrices that are distinguished only by the signs of their entries, $\{+,-, 0\}--$ has been in existence for decades ( $c f .[1,3])$, with a recently renewed interest due to the M-theory in quantum physics (see, e.g., [5]); major research interests on such "qualitative matrices" have been about the properties of positive matrices or the conditions of matrix invertibility $($ see, e.g., $[4,6])$. Our $\{H\}$ here actually contain positive matrices as a special case.

We will show that such matrices $\{H\}$ can not have eigenvalues that lie on the imaginary axis of the complex-plane, except possibly at the point of origin. Our motivation here is that, if of full rank, $\{H\}$ are then hyperbolic, which is a theme of interest in the study of dynamical systems (cf., e.g., [2] , 107).

Section 2 below will derive the proposition, and Section 3 will draw a summary. 


\section{The Proposition}

Definition 1 A matrix $H_{n \times n}$ will be termed (here in this paper by the author) "quasi-hyperbolic" if each of its eigenvalues $\lambda_{j}, j=1, \cdots, n$, satisfies $\lambda_{j}^{2} \notin$ $(-\infty, 0)$; if in addition $\lambda_{j} \neq 0, \forall j=1, \cdots, n$, then $H$ is (as defined in the textbook) hyperbolic.

Proposition 1 Assume that matrix $H_{n \times n}$ is symmetric in signs, i.e., sgnh $_{i j}=$ $\operatorname{sgnh}_{j i} \in\{1,-1,0\} \forall i \forall j \in\{1, \cdots, n\}$. Then $H_{n \times n}$ is quasi-hyperbolic.

Proof. Let $H \equiv\left(h_{i j}\right)_{n \times n}$ be as assumed. Let $\epsilon>0$, and $\hat{I} \equiv\left(\hat{\delta}_{i j}\right)_{n \times n}$ with

$$
\hat{\delta}_{i j}:=10^{-\kappa} \delta_{i j}, \kappa \equiv \frac{\delta_{1 j}}{\epsilon} \cdot(\text { Kronecker delta })
$$

Consider $\tilde{H} \equiv\left(\tilde{h}_{i j}\right):=\hat{I} H \hat{I}^{-1}$, which shares the same set of eigenvalues as $H$. Let $\mathbf{b} \equiv\left(b_{i}\right)_{n \times 1} \in \mathbb{R}^{n}$, and consider $\mathbf{e}_{1} \tilde{H}^{2} \mathbf{b}$, where $\mathbf{e}_{1} \equiv\left(\delta_{1 j}\right)_{1 \times n}$. We have:

$$
\begin{aligned}
\mathbf{e}_{1} \tilde{H}^{2} \mathbf{b} & =\left(\sum_{j=1}^{n} h_{1 j} h_{j 1}, \quad \hat{\delta}_{11} \sum_{j=1}^{n} h_{1 j} h_{j 2}, \cdots, \quad \hat{\delta}_{11} \sum_{j=1}^{n} h_{1 j} h_{j n}\right) \mathbf{b} \\
& =b_{1} \sum_{j=1}^{n} h_{1 j} h_{j 1}+\hat{\delta}_{11}\left(\sum_{i=2}^{n} b_{i} \sum_{j=1}^{n} h_{1 j} h_{j i}\right)
\end{aligned}
$$

choose $\epsilon$ and thus $\hat{\delta}_{11}$ small; since $\sum_{j=1}^{n} h_{1 j} h_{j 1} \geq 0$, we have $\operatorname{sgn}\left(\mathbf{e}_{1} \tilde{H}^{2} \mathbf{b}\right)$. $\operatorname{sgn}\left(b_{1}\right) \geq 0$; thus, $\tilde{H}^{2} \equiv \hat{I} H^{2} \hat{I}^{-1}$ has no negative eigenvalues, and hence $H$ has no eigenvalues $\pm r i \forall r>0$; i.e., $H$ is quasi-hyperbolic.

Example 1 As a simple illustration, consider the matrix

$$
\left(\begin{array}{cc}
1 & a \\
b & -1
\end{array}\right)
$$

which has eigenvalues

$$
\lambda= \pm \sqrt{1+a b}
$$

thus, $\lambda^{2}<0$ implies that $a b<0$.

Remark 1 Since $H$ has no pure imaginary eigenvalues, any continuous signpreserving rank-preserving transformation of $H$ must leave all the eigenvalues of $H$ staying on the same side of the imaginary axis on the complex-plane. As such, if $H$ represents a linearized dynamical system around an equilibrium point, then its stability properties also remain invariant under such transformations. Two particular cases here are: (1) (small) perturbation of $H$; (2) multiplication of $H$ by a positive diagonal matrix - - a standard modeling of price dynamics in economics. 


\section{Summary}

The study of qualitative matrices has been a special field in linear algebra for decades. Our paper here has contributed to the knowledge of a special class of matrices $\{H\}$; as many relations are mutual, $\{H\}$, being symmetric in signs, constitute a large class of matrix modeling in applications. Furthermore, once a matrix $H$ is ascertained to be of full rank, then it is hyperbolic - - some information that is very pertinent to dynamical systems.

\section{References}

[1] A. Brieden and S. Cokus, On the hardness of efficiently approximating maximal non-L submatrices, Linear Algebra and its Applications, 377 (2004), 195-205.

[2] J.K. Hale, Ordinary Differential Equations, Wiley-Interscience, New York, 1980.

[3] F.J. Hall, Z. Li and D. Wang, Symmetric sign pattern matrices that require unique inertia, Linear Algebra and its Applications, 338 (1) (2001), 153-169.

[4] C.R. Johnson, T.J. Laffey and R. Loewy, The real and the symmetric nonnegative inverse eigenvalue problems are different, Proc. Amer. Math. Soc. 124 (1996), 3647-3651.

[5] K. Rietsch, Totally positive Toeplitz matrices and quantum cohomology of partial flag varieties, J. Amer. Math. Soc. 16 (2003), 363-392.

[6] J.-Y. Shao and H.-Y. Shan, Matrices with signed generalized inverses, Linear Algebra and its Applications, 322 (1) (2001), 105127.

Received: September 23, 2005 